\title{
Finite Temperature and Large Gauge Invariance
}

\author{
Ashok Das \\ Department of Physics and Astronomy, \\ University of Rochester, \\ Rochester, New York, 14627
}

Received on 1 February, 2001

\begin{abstract}
In this talk, I will sumarize the status of our understanding of the puzzle of large gauge invariance
\end{abstract} at finite temperature.

\section{Introduction}

Gauge theories are beautiful theories which describe physical forces in a natural manner and because of their rich structure, the study of gauge theories at finite temperature [1] is quite interesting in itself. However, to avoid getting into technicalities, we will not discuss the intricacies of such theories either at zero temperature or at finite temperature. Rather, we would study a particular puzzle that arises when a fermion interacts with an external gauge field in odd space-time dimensions.

To motivate, let us note that gauge invariance is realized as an internal symmetry in quantum mechanical systems. Consequently, we do not expect a macroscopic external surrounding, such as a heat bath, to modify gauge invariance. This is more or less what is also found by explicit computations at finite temperature, namely, that gauge invariance and Ward identities continue to hold even at finite temperature [2]. This is certainly the case when one is talking about small gauge transformations for which the parameters of transformation vanish at infinity.

However, there is a second class of gauge transformations, commonly known as large gauge transformations, where the parameters do not vanish at infinity. In odd space-time dimensions, invariance under large gauge transformations leads to some interesting features in physical theories. Let us note that, in odd space-time dimensions, one can, in addition to the usual Maxwell (Yang-Mils) term, have a topological term in the gauge Lagrangian known as the Chern-Simons term. For example, in $2+1$ dimensions, a fermion interacting with a non-Abelian gauge field can be described by a Lagrangian density of the form

$$
\begin{aligned}
\mathcal{L} & =\mathcal{L}_{\text {gauge }}-m \mathcal{L}_{\mathrm{CS}}+\mathcal{L}_{\text {fermion }} \\
& =\frac{1}{2} \operatorname{tr} F_{\mu \nu} F^{\mu \nu}-m \epsilon^{\mu \nu \lambda} \operatorname{tr} A_{\mu}\left(\partial_{\nu} A_{\lambda}\right. \\
& \left.+\frac{2 g}{3} A_{\nu} A_{\lambda}\right)+\bar{\psi}\left(\gamma^{\mu}\left(i \partial_{\mu}-g A_{\mu}\right)-M\right) \psi
\end{aligned}
$$

where $m$ is a mass parameter, $A_{\mu}$ a matrix valued nonAbelian gauge field in a given representation and "tr" stands for the matrix trace. The first term, on the right hand side, is the usual Yang-Mills term while the second is known as the Chern-Simons term which exists only in odd space-time dimensions. It is a topological term (since it does not involve the metric) and, in the presence of a Yang-Mils term, its effect is to provide a gauge invariant mass term to the gauge fields. Consequently, such a term is also known as a topological mass term [3]. (Such a term also breaks various discrete symmetries, but we will not get into that.)

Under a gauge transformation of the form

$$
\begin{aligned}
\psi & \rightarrow U^{-1} \psi \\
A_{\mu} & \rightarrow U^{-1} A_{\mu} U-\frac{i}{g} U^{-1} \partial_{\mu} U
\end{aligned}
$$

it is straightforward to check that both the Yang-Mills and the fermion terms are invariant, while the ChernSimons term changes by a total divergence leading to

$$
S=\int d^{3} x \mathcal{L} \rightarrow S+\frac{4 \pi m}{g^{2}} 2 i \pi W
$$

where

$$
W=\frac{1}{24 \pi^{2}} \int d^{3} x \epsilon^{\mu \nu \lambda} \operatorname{tr} \partial_{\mu} U U^{-1} \partial_{\nu} U U^{-1} \partial_{\lambda} U U^{-1}
$$

is known as the winding number for the gauge transformation. It is a topological quantity which is an integer and which groups all gauge transformations into topologically distinct classes. Basically, it counts how many times the gauge transformations wrap around the sphere. For small gauge transformations, the winding number vanishes since the gauge transformations vanish at infinity whereas non-vanishing winding numbers give rise to large gauge transformations. 
Let us note from eq. (3) that even though the action is not invariant under a large gauge transformation, if $m$ is quantized in units of $\frac{g^{2}}{4 \pi}$, the change in the action would be a multiple of $2 i \pi$ and, consequently, the path integral would be invariant under a large gauge transformation. Thus, we have the constraint coming from the consistency of the theory that the coefficient of the Chern-Simons term must be quantized in units of $\frac{g^{2}}{4 \pi}$. (From an operator point of view, such a condition arises from an analysis of the dimensionality of the Hilbert space.)

We have derived the quantization of the CS coefficient from an analysis of the large gauge invariance of the tree level action and we have to worry if the quantum corrections can change the behavior of the theory. At zero temperature, an analysis of the quantum corrections shows that the theory continues to be well defined with the tree level quantization of the Chern-Simons coefficient provided the number of fermion flavors is even. The even number of fermion flavors is also necessary for a global anomaly of the theory to vanish and so, everything is well understood at zero temperature. (Namely, the quantum corrections, with an even number of fermion flavors shift the tree level integer value to another, thereby maintaining large gauge invariance.)

At finite temperature, however, the situation appears to change drastically. Namely, the fermions induce a temperature dependent Chern-Simons term leading to [4]

$$
m \rightarrow m-\frac{g^{2}}{4 \pi} \frac{M N_{f}}{2|M|} \tanh \frac{\beta|M|}{2}
$$

Here, $N_{f}$ is the number of fermion flavors and $\beta=\frac{1}{k T}$. This shows that, at zero temperature $(\beta \rightarrow \infty), m$ changes by an integer (in units of $g^{2} / 4 \pi$ ) for an even number of flavors. However, at finite temperature, the CS coefficient becomes a continuous function of temperature and, consequently, it is clear that it can no longer be an integer for arbitrary values of the temperature, as is required for large gauge invariance. It seems, therefore, that temperature would lead to a breaking of large gauge invariance in such a system. This, on the other hand, is completely counter intuitive considering that temperature should have no direct influence on gauge invariance of the theory. As a result, we are left with a puzzle, whose resolution, as we will see, is quite interesting.

\section{C-S Theory in $0+1$ Dimen- sion:}

The $2+1$ dimensional theory described in the previous section is quite complicated to carry out higher order calculations. On the other hand, as we have noted, Chern-Simons terms can exist in odd space-time dimensions. Consequently, let us try to understand this puzzle of large gauge invariance in a simple quantum mechanical theory. Let us consider a simple theory of an interacting massive fermion with an Abelian gauge field in $0+1$ dimension described by $[5,6]$

$$
L=\bar{\psi}_{j}\left(i \partial_{t}-A-M\right) \psi_{j}-\kappa A
$$

Here, $j=1,2, \cdots, N_{f}$ labels the fermion flavors. There are several things to note from this. First, we are considering an Abelian gauge field for simplicity. Second, in this simple model, the gauge field has no dynamics (in $0+1$ dimension the field strength is zero) and, therefore, we do not have to get into the intricacies of gauge theories. There is no Dirac matrix in $0+1$ dimension as well making the fermion part of the theory quite simple as well. And, finally, the Chern-Simons term, in this case, is a linear field so that we can, in fact, think of the gauge field as an auxiliary field. (Note that we have set the coupling constant to unity for simplicity.)

In spite of the simplicity of this theory, it displays a rich structure including all the properties of the $2+1$ dimensional theory that we have discussed earlier. For example, let us note that under a gauge transformation

$$
\psi_{j} \rightarrow e^{-i \lambda(t)} \psi_{j}, \quad A \rightarrow A+\partial_{t} \lambda(t)
$$

the fermion part of the Lagrangian is invariant, but the Chern-Simons term changes by a total derivative giving

$$
S=\int d t L \rightarrow S-2 \pi \kappa N
$$

where

$$
N=\frac{1}{2 \pi} \int d t \partial_{t} \lambda(t)
$$

is the winding number and is an integer which vanishes for small gauge transformations. Let us note that a large gauge transformation can have a parametric form of the form, say,

$$
\lambda(t)=-i N \log \left(\frac{1+i t}{1-i t}\right)
$$

The fact that $N$ has to be an integer can be easily seen to arise from the requirement of single-valuedness for the fermion field. Once again, in light of our earlier discussion, it is clear from eq. (8) that the theory is meaningful only if $\kappa$, the coefficient of the ChernSimons term, is an integer.

Let us assume, for simplicity, that $M>0$ and compute the correction to the photon one-point function arising from the fermion loop at zero temperature.

$$
i I_{1}=-(-i) N_{f} \int \frac{d k}{2 \pi} \frac{i(k+M)}{k^{2}-M^{2}+i \epsilon}=\frac{i N_{f}}{2}
$$

This shows that, as a result of the quantum correction, the coefficient of the Chern-Simons term would change as

$$
\kappa \rightarrow \kappa-\frac{N_{f}}{2}
$$


As in $2+1$ dimensions, it is clear that the coefficient of the Chern-Simons term would continue to be quantized and large gauge invariance would hold if the number of fermion flavors is even. At zero temperature, we can also calculate the higher point functions due to the fermions in the theory and they all vanish. This has a simple explanation following from the small gauge invariance of the theory [7]. Namely, suppose we had a nonzero two point function, then, it would imply a quadratic term in the effective action of the form

$$
\Gamma_{2}=\frac{1}{2} \int d t_{1} d t_{2} A\left(t_{1}\right) F\left(t_{1}-t_{2}\right) A\left(t_{2}\right)
$$

Furthermore, invariance under a small gauge transformation would imply

$$
\delta \Gamma_{2}=-\int d t_{1} d t_{2} \lambda\left(t_{1}\right) \partial_{t_{1}} F\left(t_{1}-t_{2}\right) A\left(t_{2}\right)=0
$$

The solution to this equation is that $F=0$ so that there cannot be a quadratic term in the effective action which would be local and yet be invariant under small gauge transformations. A similar analysis would show that small gauge invariance does not allow any higher point function to exist at zero temperature.

Let us also note that eq. (13) has another solution, namely,

$$
F\left(t_{1}-t_{2}\right)=\text { constant }
$$

In such a case, however, the quadratic action becomes non-extensive, namely, it is the square of an action. We do not expect such terms to arise at zero temperature and hence the constant has to vanish for vanishing temperature. As we will see next, the constant does not have to vanish at finite temperature and we can have non-vanishing higher point functions implying a non-extensive structure of the effective action.

The fermion propagator at finite temperature (in the real time formalism) has the form [1]

$$
\begin{aligned}
S(p) & =(p+M)\left(\frac{i}{p^{2}-M^{2}+i \epsilon}-2 \pi n_{F}(|p|) \delta\left(p^{2}-M^{2}\right)\right) \\
& =\frac{i}{p-M+i \epsilon}-2 \pi n_{F}(M) \delta(p-M)
\end{aligned}
$$

and the structure of the effective action can be studied in the momentum space in a straightforward manner. However, in this simple model, it is much easier to analyze the amplitudes in the coordinate space. Let us note that the coordinate space structure of the fermion propagator is quite simple, namely,

$$
S(t)=\int \frac{d p}{2 \pi} e^{-i p t}\left(\frac{i}{p-M+i \epsilon}-2 \pi n_{F}(M) \delta(p-M)\right)=\left(\theta(t)-n_{F}(M)\right) e^{-i M t}
$$

In fact, the calculation of the one point function is trivial now

$$
i I_{1}=-(-i) N_{f} S(0)=\frac{i N_{f}}{2} \tanh \frac{\beta M}{2}
$$

This shows that the behavior of this theory is completely parallel to the $2+1$ dimensional theory in that, it would suggest

$$
\kappa \rightarrow \kappa-\frac{N_{f}}{2} \tanh \frac{\beta M}{2}
$$

and it would appear that large gauge invariance would not hold at finite temperature.

Let us next calculate the two point function at finite temperature.

$$
\begin{aligned}
i I_{2} & =-(-i)^{2} \frac{N_{f}}{2 !} S\left(t_{1}-t_{2}\right) S\left(t_{2}-t_{1}\right) \\
& =-\frac{N_{f}}{2} n_{F}(M)\left(1-n_{F}(M)\right) \\
& =-\frac{N_{f}}{8} \operatorname{sech}^{2} \frac{\beta M}{2}=\frac{1}{2} \frac{1}{2 !} \frac{i}{\beta} \frac{\partial\left(i I_{1}\right)}{\partial M}
\end{aligned}
$$

This shows that the two point function is a constant as we had noted earlier implying that the quadratic term in the effective action would be non-extensive.

Similarly, we can also calculate the three point function trivially and it has the form 


$$
i I_{3}=\frac{i N_{f}}{24} \tanh \frac{\beta M}{2} \operatorname{sech}^{2} \frac{\beta M}{2}=\frac{1}{2} \frac{1}{3 !}\left(\frac{i}{\beta}\right)^{2} \frac{\partial^{2}\left(i I_{1}\right)}{\partial M^{2}}
$$

In fact, all the higher point functions can be worked out in a systematic manner. But, let us observe a simple method of computation for these. We note that because of the gauge invariance (Ward identity), the amplitudes cannot depend on the external time coordinates as is clear from the calculations of the lower point functions. Therefore, we can always simplify the calculation by choosing a particular time ordering convenient to us. Second, since we are evaluating a loop diagram (a fermion loop) the initial and the final time coordinates are the same and, consequently, the phase factors in the propagator (15) drop out. Therefore, let us define a simplified propagator without the phase factor as

$$
\widetilde{S}(t)=\theta(t)-n_{F}(M)
$$

so that we have

$$
\widetilde{S}(t>0)=1-n_{F}(M), \quad S(t<0)=-n_{F}(M)
$$

Then, it is clear that with the choice of the time ordering, $t_{1}>t_{2}$, we can write

$$
\begin{array}{ll}
\frac{\partial \widetilde{S}\left(t_{1}-t_{2}\right)}{\partial M}=-\beta \widetilde{S}\left(t_{1}-t_{3}\right) \widetilde{S}\left(t_{3}-t_{2}\right) & t_{1}>t_{2}>t_{3} \\
\frac{\partial \widetilde{S}\left(t_{2}-t_{1}\right)}{\partial M}=-\beta \widetilde{S}\left(t_{2}-t_{3}\right) \widetilde{S}\left(t_{3}-t_{1}\right) & t_{1}>t_{2}>t_{3}
\end{array}
$$

In other words, this shows that differentiation of a fermionic propagator with respect to the mass of the fermion is equivalent to introducing an external photon vertex (and, therefore, another fermion propagator as well) up to constants. This is the analogue of the Ward identity in QED in four dimensions except that it is much simpler. From this relation, it is clear that if we take a $n$-point function and differentiate this with respect to the fermion mass, then, that is equivalent to adding another external photon vertex in all possible positions. Namely, it should give us the $(n+1)$-point function up to constants. Working out the details, we have,

$$
\frac{\partial I_{n}}{\partial M}=-i \beta(n+1) I_{n+1}
$$

Therefore, the $(n+1)$-point function is related to the $n$-point function recursively and, consequently, all the amplitudes are related to the one point function which we have already calculated. (Incidentally, this is already reflected in eqs. $(17,18))$.

With this, we can now determine the full effective action of the theory at finite temperature to be

$$
\begin{aligned}
\Gamma & =-i \sum_{n} a^{n}\left(i I_{n}\right) \\
& =-\frac{i \beta N_{f}}{2} \sum_{n} \frac{(i a / \beta)^{n}}{n !}\left(\frac{\partial}{\partial M}\right)^{n-1} \tanh \frac{\beta M}{2} \\
& =-i N_{f} \log \left(\cos \frac{a}{2}+i \tanh \frac{\beta M}{2} \sin \frac{a}{2}\right)
\end{aligned}
$$

where we have defined

$$
a=\int d t A(t)
$$

There are several things to note from this result. First of all, the higher point functions are no longer vanishing at finite temperature and give rise to a nonextensive structure of the effective action. More importantly, when we include all the higher point functions, the complete effective action is invariant under large gauge transformations, namely, under

$$
a \rightarrow a+2 \pi N
$$

the effective action changes as

$$
\Gamma \rightarrow \Gamma+N N_{f} \pi
$$

which leaves the path integral invariant for an even number of fermion flavors. This clarifies the puzzle of large gauge invariance at finite temperature in this model. Namely, when we are talking about large changes (large gauge transformations), we cannot ignore higher order terms if they exist. This may provide a resolution to the large gauge invariance puzzle in the $2+1$ dimensional theory as well.

\section{Exact Result:}

In the earlier section, we discussed a perturbative method of calculating the effective action at finite tem- 
perature which clarified the puzzle of large gauge invariance. However, this quantum mechanical model is simple enough that we can also evaluate the effective action directly and, therefore, it is worth asking how the perturbative calculations compare with the exact result.

The exact evaluation of the effective action can be done easily using the imaginary time formalism. But, first, let us note that the fermionic part of the Lagrangian in eq. (6) has the form

$$
L_{f}=\bar{\psi}\left(i \partial_{t}-A-M\right) \psi
$$

where we have suppressed the fermion flavor index for simplicity. Let us note that if we make a field redefinition of the form

$$
\psi(t)=e^{-i \int_{0}^{t} d t^{\prime} A\left(t^{\prime}\right)} \tilde{\psi}(t)
$$

then, the fermionic part of the Lagrangian becomes free, namely,

$$
L_{f}=\overline{\tilde{\psi}}\left(i \partial_{t}-M\right) \tilde{\psi}
$$

This is a free theory and, therefore, the path integral can be easily evaluated. However, we have to remember that the field redefinition in (28) changes the periodicity condition for the fermion fields. Since the original fermion field was expected to satisfy anti-periodicity

$$
\psi(\beta)=-\psi(0)
$$

it follows now that the new fields must satisfy

$$
\tilde{\psi}(\beta)=-e^{-i a} \tilde{\psi}(0)
$$

Consequently, the path integral for the free theory (29) has to be evaluated subject to the periodicity condition of $(30)$.

Although the periodicity condition (30) appears to be complicated, it is well known that this can be absorbed by introducing a chemical potential [1], in the present case, of the form

$$
\mu=\frac{i a}{\beta}
$$

With the addition of this chemical potential, the path integral can be evaluated subject to the usual antiperiodicity condition. The effective action can now be easily determined

$$
\begin{aligned}
\Gamma & =-i \log \left(\frac{\operatorname{det}\left(i \partial_{t}-M+\frac{i a}{\beta}\right)}{\left(i \partial_{t}-M\right)}\right)^{N_{f}} \\
& =-i N_{f} \log \left(\frac{\cosh \frac{\beta}{2}\left(M-\frac{i a}{\beta}\right)}{\cosh \frac{\beta M}{2}}\right) \\
& =-i N_{f} \log \left(\cos \frac{a}{2}+i \tanh \frac{\beta M}{2} \sin \frac{a}{2}\right)
\end{aligned}
$$

which coincides with the perturbative result of eq. (24).

\section{Large Gauge Ward Identity:}

It is clear from the above analysis that, to see if large gauge invariance is restored, we have to look at the complete effective action. In the $0+1$ dimensional model, it was tedious, but we can derive the effective action in closed form which allows us to analyze the question of large gauge invariance. On the other hand, in the theory of interest, namely, the $2+1$ dimensional Chern-Simons theory, we do not expect to be able to evaluate the effective action in a closed form. Consequently, we must look for an alternate way to analyze the question of large gauge invariance in a more realistic model. One such possible method may be to derive a Ward identity for large gauge invariance which will relate different amplitudes much like the Ward identity for small gauge invariance does. In such a case, even if we cannot obtain the effective action in a closed form, we can at least check if the large gauge Ward identity holds perturbatively.

It turns out that the large gauge Ward identities are highly nonlinear [8], as we would expect. Hence, looking for them within the context of the effective action is extremely hard (although it can be done). Rather, it is much simpler to look at the large gauge Ward identities in terms of the exponential of the effective action. Let us define

$$
\Gamma(a)=-i \log W(a)
$$

Namely, we are interested in looking at the exponential of the effective action (i.e. up to a factor of $i, W$ is the basic determinant that would arise from integrating out the fermion field). We will restrict ourselves to a single flavor of massive fermions. The advantage of studying $W(a)$ as opposed to the effective action lies in the fact that, in order for $\Gamma(a)$ to have the right transformation properties under a large gauge transformation, $W(a)$ simply has to be quasi-periodic. Consequently, from the study of harmonic oscillator (as well as Floquet theory), we see that $W(a)$ has to satisfy a simple equation of the form

$$
\frac{\partial^{2} W(a)}{\partial a^{2}}+\nu^{2} W(a)=g
$$

where $\nu$ and $g$ are parameters to be determined from the theory. In particular, let us note that the constant $g$ can depend on parameters of the theory such as temperature whereas we expect the parameter $\nu$, also known as the characteristic exponent, to be independent of temperature and equal to an odd half integer for a fermionic mode. However, all these properties should automatically result from the structure of the theory. Let us also note here that the relation (34) is simply the equation for a forced oscillator whose solution has the general form 


$$
W(a)=\frac{g}{\nu^{2}}+A \cos (\nu a+\delta)=\frac{g}{\nu^{2}}+\alpha_{1} \cos \nu a+\alpha_{2} \sin \nu a
$$

The constants $\alpha_{1}$ and $\alpha_{2}$ appearing in the solution can again be determined from the theory. Namely, from the relation between $W(a)$ and $\Gamma(a)$, we recognize that we can identify

$$
\begin{aligned}
\nu^{2} \alpha_{1} & =-\left.\frac{\partial^{2} W}{\partial a^{2}}\right|_{a=0}=\left.\left(\left(\frac{\partial \Gamma}{\partial a}\right)^{2}-i \frac{\partial^{2} \Gamma}{\partial a^{2}}\right)\right|_{a=0} \\
\nu \alpha_{2} & =\left.\frac{\partial W}{\partial a}\right|_{a=0}=\left.i \frac{\partial \Gamma}{\partial a}\right|_{a=0}
\end{aligned}
$$

From the general properties of the fermion theories we have discussed, we intuitively expect $g=0$. However, these should really follow from the structure of the theory and they do, as we will show shortly.

The identity (34) is a linear relation as opposed to the Ward identity in terms of the effective action. In fact, rewriting this in terms of the effective action (using eq. (33)), we have

$$
\frac{\partial^{2} \Gamma(a)}{\partial a^{2}}=i\left(\nu^{2}-\left(\frac{\partial \Gamma(a)}{\partial a}\right)^{2}\right)-i g e^{-i \Gamma(a)}
$$

So, let us investigate this a little bit more in detail. We know that the fermion mass term breaks parity and, consequently, the radiative corrections would generate a Chern-Simons term, namely, in this theory, we expect the one-point function to be nonzero. Consequently, by taking derivative of eq. (37) (as well as remembering that $\Gamma(a=0)=0)$, we determine (The superscript represents the number of flavors.)

$$
\begin{aligned}
\left(\nu^{(1)}\right)^{2} & =\left[\left(\frac{\partial \Gamma^{(1)}}{\partial a}\right)^{2}-3 i \frac{\partial^{2} \Gamma^{(1)}}{\partial a^{2}}-\left(\frac{\partial \Gamma^{(1)}}{\partial a}\right)^{-1}\left(\frac{\partial^{3} \Gamma^{(1)}}{\partial a^{3}}\right)\right]_{a=0} \\
g^{(1)} & =-\left[2 i \frac{\partial^{2} \Gamma^{(1)}}{\partial a^{2}}+\left(\frac{\partial \Gamma^{(1)}}{\partial a}\right)^{-1}\left(\frac{\partial^{3} \Gamma^{(1)}}{\partial a^{3}}\right)\right]_{a=0}
\end{aligned}
$$

This is quite interesting, for it says that the two parameters in eq. (34) or (37) can be determined from a perturbative calculation. Let us note here some of the perturbative results in this theory, namely,

$$
\begin{aligned}
\left.\frac{\partial \Gamma^{(1)}}{\partial a}\right|_{a=0} & =\frac{1}{2} \tanh \frac{\beta M}{2} \\
\left.\frac{\partial^{2} \Gamma^{(1)}}{\partial a^{2}}\right|_{a=0} & =\frac{i}{4} \operatorname{sech}^{2} \frac{\beta M}{2} \\
\left.\frac{\partial^{3} \Gamma^{(1)}}{\partial a^{3}}\right|_{a=0} & =\frac{1}{4} \tanh \frac{\beta M}{2} \operatorname{sech}^{2} \frac{\beta M}{2}
\end{aligned}
$$

Using these, we immediately determine from eq. (38) that

$$
\left(\nu^{(1)}\right)^{2}=\frac{1}{4}, \quad g^{(1)}=0
$$

so that the equation (37) leads to the large gauge Ward identity for a single fermion theory of the form,

$$
\frac{\partial^{2} \Gamma^{(1)}}{\partial a^{2}}=i\left(\frac{1}{4}-\left(\frac{\partial \Gamma^{(1)}}{\partial a}\right)^{2}\right)
$$

Furthermore, we determine now from eq. (36)

$$
\alpha_{1}^{(1)}=1, \quad \alpha_{2}^{(1)}= \pm i \tanh \frac{\beta M}{2}
$$

The two signs in of $\alpha_{2}^{(1)}$ simply corresponds to the two possible signs of $\nu^{(1)}$. With this then, we can solve for $W(a)$ in the single flavor fermion theory and we have (independent of the sign of $\nu^{(1)}$ )

$$
W_{f}^{(1)}(a)=\cos \frac{a}{2}+i \tanh \frac{\beta M}{2} \sin \frac{a}{2}
$$

which can be compared with eq. (32). For $N_{f}$ flavors, similarly, we can determine the Ward identity to be

$$
\frac{\partial \Gamma^{\left(N_{f}\right)}}{\partial a^{2}}=i N_{f}\left(\frac{1}{4}-\frac{1}{N_{f}^{2}}\left(\frac{\partial \Gamma^{\left(N_{f}\right)}}{\partial a^{2}}\right)^{2}\right)
$$

where the nonlinearity of the Ward identity is manifest.

Similarly, we can determine the large gauge Ward identity for scalar theories as well as supersymmetric theories, but we will not go into the details of this. 


\section{Back to $2+1$ dimensions:}

The analysis of various $0+1$ dimensional models shows [9] how the large gauge invariance puzzle gets resolved. A crucial feature in this was the existence of nonextensive higher order terms in the effective action. To further understand this feature, we have also studied the effective action for a fermion interacting with an external gauge field in $1+1$ dimensions [10]. In that case, the effective action is non-local, as it should be at finite temperature, but extensive. Furthermore, the effective action shows non-analyticity, as one would expect at finite temperature, unlike the $0+1$ dimensional model, where one does not expect any non-analyticity.

Following the results of the $0+1$ dimensional model, it was shown [11] that, for the special choice of the gauge field backgrounds where $A_{0}=A_{0}(t)$ and $\vec{A}=$ $\vec{A}(\vec{x})$, the parity violating part of the effective action of a fermion interacting with an Abelian gauge field takes the form

$$
\Gamma^{P V}=\frac{i e}{2 \pi} \int d^{2} x \arctan \left(\tanh \frac{\beta M}{2} \tan \left(\frac{e a}{2}\right)\right) B(\vec{x})
$$

However, because the choice of the background is very special, it would seem that this may not represent the complete effective action in a general background. In fact, in higher dimensions, such as $2+1$, one also has to tackle with the question of the non-analyticity of the thermal amplitudes which leads to a nonuniqueness of the effective action $[1,12]$.

With these issues in mind, we have studied the parity violating part of the four point function in $2+1$ dimensions at finite temperature. The calculations are clearly extremely difficult and we have evaluated the amplitudes at finite temperature by using the method of forward scattering amplitudes [13]. Without going into details, let me summarize the results here [14]. First, the parity violating part of the box diagram is nontrivial at zero temperature and comes from an effective action of the form

$$
\Gamma_{T=0}^{4}=-\frac{e^{4}}{64 \pi M^{6}} \int d^{3} x \epsilon^{\mu \nu \lambda} F_{\mu \nu}\left(\partial^{\tau} F_{\tau \lambda}\right) F^{\rho \sigma} F_{\rho \sigma}
$$

This is Lorentz invariant and is invariant under both small and large gauge transformations and is compatible with the Coleman-Hill theorem [15].

At finite temperature, however, the amplitude is not manifestly Lorentz invariant (because of the heat bath) and is non-analytic. We have investigated the amplitude in two interesting limits. Namely, in the long wave limit (all spatial momenta vanishing), the leading term of the amplitude, at high temperature, can be seen to come from an effective action of the form

$\Gamma_{L W}^{4}=\frac{e^{4}}{512 M T} \int d^{3} x \epsilon_{0 i j} E_{i}\left(\partial_{t}^{-1} E_{j}\right)\left(\partial_{t}^{-1} E_{k}\right)\left(\partial_{t}^{-1} E_{k}\right)$ where $\vec{E}$ represents the electric field. There are several things to note here. First, this is an extensive action, be it non-local. Second, it is manifestly large gauge invariant and finally, the leading behavior at high temperature goes as $\frac{1}{T}$.

In contrast, we can evaluate the amplitude in the static limit (all energies vanishing) where we find the presence of both extensive as well as non-extensive terms at high temperature. However, the extensive terms are suppressed by powers of $T$ and the leading term seems to come from an effective action of the form

$$
\Gamma_{S}^{4}=\frac{e^{4}}{4 \pi T^{2}}\left(\tanh \frac{\beta M}{2}-\tanh ^{3} \frac{\beta M}{2}\right) \int d^{3} x a^{3} B
$$

This coincides with the amplitude that will come from eq. (45) and has the leading behavior of $\frac{1}{T^{3}}$ at high temperature. Such a term is not invariant under a large gauge transformation. However, we can now derive a large gauge Ward identity for the leading part of the static action and the solution of the Ward identity coincides with the form given in eq. (45). This, therefore, clarifies the meaning of the effective action in the special background, namely, it represents the leading term in the effective action in the static limit.

\section{Higher order corrections:}

Since we have calculated the box diagram at finite temperature, we can also ask about possible higher loop corrections to the CS coefficient. Let us note that, at zero temperature, there is a result due to Coleman and Hill [15], which says that in an Abelian theory, there cannot be any correction to the CS coefficient beyond one loop. Their result basically uses two simple assumptions, i) small gauge invariance and ii) analyticity of the amplitudes in the momentum space. Small gauge invariance is, of course, known to be true at finite temperature. However, as we have pointed out earlier, amplitudes become non-analytic in the momentum space at finite temperature. Therefore, the second assumption of Coleman-Hill breaks down at finite temperature and one may expect higher loop correction to the CS coefficient at finite temperature.

We have explicitly computed the two loop correction to the CS coefficient at finite temperature [16]. Parameterizing the self-energy in a covariant gauge as

$$
\Pi^{\mu \nu}(p, u)=\Pi_{1}^{\mu \nu}(p, u)+i \epsilon^{\mu \nu \lambda} p_{\lambda} \Pi_{2}(p, u)
$$

where $u^{\mu}$ represents the velocity of the heat bath, we find that, in the static limit, the two loop correction to the CS coefficient at high temperatures takes the form

$$
\Pi_{2}^{(2)}(0)=(2 m-3 M) \frac{e^{4}}{192 \pi^{2} T^{2}} \ln \frac{T}{m}
$$


This result explicitly shows that the Coleman-Hill result breaks down at finite temperatures. Furthermore, the form of the correction is interesting in that it diverges as $m \rightarrow 0$. This is the usual manifestation of infrared divergence and shows that, although the zero temperature theory is well defined in the limit of vanishing $m$, there are infrared divergences at finite temperature. In addition, since there is a two loop correction to the CS coefficient, one may ask the structure of the effective action when higher order corrections are taken into account. This can be determined from the large gauge Ward identity that we have derived and it determines the form of the parity violating effective action, satisfying large gauge invariance, at any order to be

$$
\Gamma^{P V}=\tan ^{-1}\left(2 \Gamma^{\prime}(0) \tan \frac{e a}{2}\right) \int d^{2} x B
$$

where $\Gamma^{\prime}(0)$ represents the correction to the CS coefficient to that order.

\section{Non-Abelian theories:}

Our main interest was in the study of the question of large gauge invariance at finite temperature in a nonAbelian theory. As we have shown, the question of large gauge invariance is well understood in an Abelian theory. However, not much is known about the nonAbelian theory yet. To that extent, let us note that even the Coleman-Hill result was derived for an Abelian theory. In this section, let me describe briefly how the Coleman-Hill result can be generalized to non-Abelian theories (at zero temperature).

There are several qualitative differences between the Abelian and the non-Abelian theories. First, while the Abelian theory is well defined even when the tree level CS coefficient vanishes, the infrared divergences in the non-Abelian theory are severe if there is no tree level CS term. Second, while the Abelian theory can be defined in any gauge, the non-Abelian theory is well defined only in a select class of infrared safe gauges such as the Landau gauge, the axial gauge etc. Finally, in the Abelian theory, the CS coefficient is a gauge independent quantity while in the non-Abelian theory, the CS coefficient is gauge dependent. As a result, it is not clear how to generalize the Coleman-Hill result in the non-Abelian case.

On the other hand, it is known from various arguments that, in a non-Abelian theory, it is the ratio $\frac{4 \pi m}{g^{2}}$ which has a physical meaning and, therefore, must be gauge independent. In fact, a one loop calculation verifies that in all infrared safe gauges the one loop correction to this ratio is given by

$$
\frac{4 \pi m}{g^{2}} \rightarrow \frac{4 \pi m}{g^{2}}+N
$$

It is, therefore, meaningful to generalize the ColemanHill result for this ratio. (Recall that it is also this ratio that needs to be quantized for large gauge invariance to hold.)

This can indeed be done as follows [17]. First, let us note that, although the CS coefficient is gauge dependent in general, it takes on a physical meaning in the axial gauge. This is because in the axial gauge,

$$
\frac{4 \pi m}{g^{2}} \rightarrow \frac{4 \pi m}{g^{2}}\left(1+\Pi_{2}(0)\right)
$$

Since this ratio is physical, in this gauge $\Pi_{2}(0)$ does carry a physical meaning. Using the Ward identities in the axial gauge, it is straight forward to show that the CS coefficient does not receive any corrections beyond one loop provided i) small aguge invariance holds and ii) amplitudes are analytic in the momentum space. A consequence of this is that the ratio $\frac{4 \pi m}{g^{2}}$ does not have any correction beyond one loop in this gauge. However, this is a gauge independent quantity and hence it holds in any gauge that this ratio does not receive any correction beyond one loop. Thus, we understand some features of the zero temperature non-Abelian theory which are parallel to the Abelian theory and what remains is to understand systematically if the issue of large gauge invariance also gets resolved in a parallel manner.

\section{Conclusion:}

In this talk, we have discussed the question of large gauge invariance at finite temperature. We have discussed the resolution of the problem in a simple $0+1$ dimensional model. We have derived the Ward identity for large gauge invariance in this model. We have analyzed the box diagram in $2+1$ dimensions and have obtained the form of the effective action at zero temperature. We have also obtained the amplitude as well as the quartic effective actions in the long wave as well as static limits, at finite temperature. The LW limit has only extensive terms in the action which goes as $\frac{1}{T}$ at high temperature and is invariant under large gauge transformations. The leading term in the static action, however, is non-extensive, goes as $\frac{1}{T^{3}}$ at high temperature and coincides with the effective action proposed earlier for a restrictive gauge background. This action is not invariant under large gauge transformations. However, using a large gauge Ward identity, we can determine the full leading order action in the static limit which coincides with the effective action obtained in a restrictive gauge background. We have shown explicitly that higher loop corrections to the CS coefficient do not vanish at finite temperature. This violation of the Coleman-Hill result is a consequence of the fact that one of their assumptions (namely, analyticity of the amplitudes) breaks down at finite temperature. We have also extended the result of Coleman-Hill to the case of non-Abelian gauge theories. 
It is a plaesure to thank the organizers for their hospitality. I have learnt a lot from all of my collaborators in the field, namely, K. Babu, J. Barcelos-Neto, F. Brandt, A. J. da Silva, G. Dunne, J. Frenkel, P. Panigrahi, K. Rao and J. C. Taylor, and I am grateful to all of them. This work was supported in part by the U.S. Dept. of Energy Grant DE-FG 02-91ER40685.

\section{References}

[1] See for example, A. Das, Finite Temperature Field Theory, World Scientific, 1997.

[2] A. Das and M. Hott, Mod. Phys. Lett. A 9, 3383 (1994).

[3] S. Deser, R. Jackiw and S. Templeton, Ann. Phys. 140, 372 (1982).

[4] K. S. Babu, A. Das and P. Panigrahi, Phys. Rev. D 36, 3725 (1987); I. Aitchison and J. Zuk, Ann. Phys. 242, 77 (1995); N. Bralić, C. Fosco and F. Schaposnik, Phys. Lett.B 383, 199 (1996); D. Cabra, E. Fradkin, G. Rossini and F. Schaposnik, Phys. Lett. B 383, 434 (1996).

[5] G. Dunne, R. Jackiw and C. Trugenberger, Phys. Rev. D 41, 661 (1990).

[6] G. Dunne, K. Lee and C. Lu, Phys. Rev. Lett. 78, 3434 (1997).

[7] A. Das and G. Dunne, Phys. Rev. D 57, 5023 (1998).
[8] A. Das, G. Dunne and J. Frenkel, Phys. Lett. B 472, $332(2000)$.

[9] J. Barcelos-Neto and A. Das, Phys. Rev. D 58, 085022 (1998); J. Barcelos-Neto and A. Das, Phys. Rev.D 59 087701 (1999); A. Das and G. Dunne, Phys. Rev. D 60, 085010 (1999).

[10] A. Das and A. J. da Silva, Phys. Rev.D 59, 105011 (1999).

[11] S. Deser, L. Griguolo and D. Seminara, Phys. Rev. Lett. 79, 1976 (1997); C. Fosco, G. Rossini and F. Schaposnik, Phys. Rev. 79, 1980 (1997); S. Deser, L. Griguolo and D. Seminara, Phys. Rev. D 57, 7444 (1998); C. Fosco, G. Rossini and F. Schaposnik, Phys. Rev. D 56, 6547 (1997).

[12] A. Das and M. Hott, Phys. Rev. D 50, 6655 (1994).

[13] J. Frenkel and J. C. Taylor, Nuc. Phys. B 374, 156 (1992); F. T. Brandt and J. Frenkel, Phys. Rev. D56, 2453 (1997).

[14] F. T. Brandt, A. Das and J. Frenkel, Phys. Rev. D 62, 085012 (2000); F. T. Brandt, A. Das and J. Frenkel, hep-ph/0004195.

[15] S. Coleman and B. Hill, Phys. Lett.B 159, 184 (1985).

[16] F. T. Brandt, A. Das, J. Frenkel and K. Rao, Phys. Lett. B 492, 393 (2000).

[17] F. T. Brandt, A. Das and J. Frenkel, Phys. Lett. B494, 339 (2000); F. T. Brandt, A. Das and J. Frenkel, hepth/0012087. 